\title{
A Q4/Q4 continuum structural topology optimization implementation
}

\author{
S.F. Rahmatalla and C.C. Swan
}

\begin{abstract}
A node-based design variable implementation for continuum structural topology optimization in a finite element framework is presented and its properties are explored in the context of solving a number of different design examples. Since the implementation ensures $\mathrm{C}^{0}$ continuity of design variables, it is immune to elementwise checkerboarding instabilities that are a concern with element-based design variables. Nevertheless, in a subset of design examples considered, especially those involving compliance minimization with coarse meshes, the implementation is found to introduce a new phenomenon that takes the form of "layering" or "islanding" in the material layout design. In the examples studied, this phenomenon disappears with mesh refinement or the enforcement of sufficiently restrictive design perimeter constraints, the latter sometimes being necessary in design problems involving bending to ensure convergence with mesh refinement. Based on its demonstrated performance characteristics, the authors conclude that the proposed nodebased implementation is viable for continued usage in continuum topology optimization.
\end{abstract}

Key words stability, checkerboarding, design convergence

\section{1}

\section{Introduction}

Continuum structural topology optimization is an increasingly powerful design tool that can be used to ob-

Received: 27 June 2002

Revised manuscript received: 11 May 2003

Published online: 1 April 2004

(C) Springer-Verlag 2004

S.F. Rahmatalla and C.C. Swan

Department of Civil and Environmental Engineering, Center for Computer-Aided Design, The University of Iowa, Iowa City, Iowa 52242, USA

e-mail: colby-swan@uiowa.edu tain optimal arrangements of structural materials to comprise mechanical systems. Since their introduction well over a decade ago (Bendsøe and Kikuchi 1988) continuum topology optimization methods have been successfully used in numerous applications, including the design of structures for minimal elastic and inelastic compliance, the design of linear elastic structures for tailored vibrational eigenvalues, and the design of systems with compliant mechanisms (for examples: Swan and Kosaka 1997; Pedersen 2000; Bruns and Tortorelli 1998; Buhl et al. 2000; Pedersen et al. 2001; and the numerous works cited in these references). In spite of these many applications and advances, numerical instabilities involving checkerboarding and mesh-dependent design solutions have persisted over a considerable period. Accordingly, many works, as cited in the review by Eschenauer and Olhoff (2001), have been published to understand better and eliminate these instabilities.

In implementing continuum structural topology optimization formulations, there is a good deal of flexibility in selecting interpolation function spaces for displacement solution fields and design variable fields. Eight of the feasible possibilities for the design of mechanical systems in two dimensions using quadrilateral finite elements are shown in Fig. 1. Of these eight possibilities, the Q4/U implementation appears most widely used to date with the nodal displacements interpolated by bilinear shape functions, and the design variables uniform on each element domain. One of the problems with the Q4/U implementation is that it does not ensure $\mathrm{C}^{0}$ continuity of the design variable field. A related problem that occurs frequently in the application of Q4/U implementations is layout distributions that oscillate from solid to void across element boundaries, forming what resemble "checkerboarding" or "chessboarding" patterns.

Diaz and Sigmund (1995) discussed the reasons for the formation of checkerboard patterns in Q4/U implementations and demonstrated that solid and void materials arranged in element-wise checkerboard patterns on meshes of uniform bilinear finite elements appear artificially stiff. Hence, in material layout design problems for which the objective is to achieve material arrangements of minimal structural compliance with respect to specified loading conditions, "checkerboarding" material arrange- 


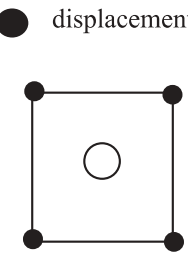

(a) $\mathrm{Q} 4 / \mathrm{U}$

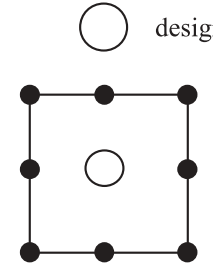

(b) $\mathrm{Q} 8 / \mathrm{U}$ design variable

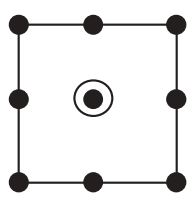

(c) Q9/U

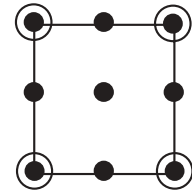

(f) $\mathrm{Q} 9 / \mathrm{Q} 4$

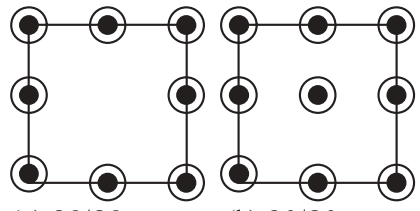

(g) Q8/Q8 (h) Q9/Q9

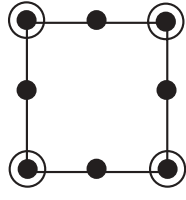

(e) Q8/Q4

Fig. 1 Selected option for element-wise interpolation of displacement fields and design variable fields

ments in the Q4/U implementation are quite natural. Later, Sigmund and Petersson (1998) surveyed many of the numerical instability problems encountered in continuum topology optimization as well as some of the common techniques for realizing non-checkerboarding material layout designs, including the usage of higher order finite elements together with element-wise uniform design variables (for example the Q8/U and Q9/U implementations in Fig. 1); the imposition of perimeter constraints; and spatial filtering of design variables. Among these options, various filtering techniques such those proposed in (Sigmund and Petersson 1998; Swan and Kosaka 1997) are widely used. However, spatial filtering of design variables is heuristic, forcing the designer to select a filter strength or filtering length scale for each design problem. Since there can be an optimal design associated with each filter strength, the design obtained will strongly depend on the filter characteristics.

Jog and Haber (1996) performed an extensive and noteworthy study on the stability of finite element solutions of distributed parameter topology optimization problems under numerous combinations of interpolation function spaces for design variables and displacement solutions. Their analysis and stability criterion found both the Q4/U and Q4/Q4 implementations to be unstable, whereas it found the $\mathrm{Q} 9 / \mathrm{U}$ (quadrilateral nine-node biquadratic displacement solution interpolation with piecewise uniform design variable interpolation) and $\mathrm{Q} 8 / \mathrm{U}$ (quadrilateral eight-node biquadratic serendipity displacement solution interpolation) implementations to be stable. In the same article, Jog and Haber mentioned, however, that checkerboarding solutions had appeared in limited cases even with the Q9/U and Q8/U implementations. This was confirmed in subsequent observations reported by Sigmund and Petersson (1998) and Swan et al. (1998) who also found mild checkerboarding to occur with the Q8/U and Q9/U implementations.

Poulsen (2002) presented a noteworthy study that considered a continuum topology implementation quite different from any of those shown in Fig. 1. In particular,

in a bilinear finite element framework (Q4 interpolation of displacements), it addressed the checkerboarding problem using wavelet basis functions to interpolate the design field. The length scales of the wavelet basis functions were not directly coupled to the finite element mesh used for analysis. By imposing certain restrictions and dealing with overlapping bases, it was shown that the method can achieve designs that are nearly free of checkerboarding.

Still another recognized strategy for eliminating checkerboarding instabilities in material layout designs is through the imposition of constraints on the design solution that limit the "fineness" of the design. For example, enforcement of sufficiently restrictive perimeter constraints (Haber et al. 1996) can be used both to preclude checkerboarding designs while also achieving designs that are convergent with mesh refinement. In a similar fashion, Fujii and Kikuchi (2000) introduced a gravity control function that they used to augment the structural compliance function. By maximizing the gravity control function, they demonstrated that checkerboards and intermediate densities are eliminated. While both perimeter and gravity-control methods are indeed effective, they require the designer to choose control values (perimeter constraint value and/or the weighting factor for the gravity control function) that strongly affect the nature of the design that will be obtained.

From this brief review it is evident that, even at this date, continuum topology optimization implementations that are immune to checkerboarding instabilities are still in demand. The intent of this article is to reexamine the Q4/Q4 implementation, as it is the lowest order implementation based on quadrilateral elements that would appear to preclude the possibility of elementwise checkerboarding design solutions by virtue of the $\mathrm{C}^{0}$ continuity of the design variable interpolation functions. Although the previously cited analysis of Jog and Haber (1996) indicated the Q4/Q4 implementation to be unstable, the nature of the potential instabilities was not elucidated therein. The limited goal of this note is to test the Q4/Q4 implementation on a reasonably broad set of continuum topology optimization problems to determine the conditions under which irregularities or instabilities of the material layout designs occur, and should they occur, the nature of the instabilities. Although attention is confined here to design examples in two spatial dimensions, the natural extension of the Q4/Q4 implementation to three dimensions would be H8/H8 implementations in which both the displacement field and design variables are interpolated with trilinear hexahedral basis functions.

In the remainder of this note, continuum topology optimization of structures with a Q4/Q4 implementation is briefly explored on a number of problems involving compliance minimization of the $\mathrm{MBB}$ beam problem for a range of mesh resolutions; the canyon bridge problem for both compliance minimization and buckling stability maximization; and the design of a sparse structure for maximum stability. A summary and discussion of the per- 
formance characteristics observed are then presented in the final section.

\section{2}

\section{Demonstrative examples}

The performance of the proposed node-based (Q4/Q4) design implementation is tested here on a number of problems that have previously been used as test problems for continuum topology optimization. In all examples, the optimization solution algorithms employed are variations of sequential linear programming (SLP) with a default LP sub-problem move limit of 0.05. A so-called "density" formulation is employed together with a power-law mixing rule with an exponent of $p=4$ (Bendsøe 1989). Unless specifically stated otherwise, the solid structural material in all design cases considered is isotropic with a Young's modulus of $307 \mathrm{GPa}$, a shear modulus of $118 \mathrm{GPa}$, and a mass density of $1700 \mathrm{~kg} \mathrm{~m}^{-3}$. Both the stiffness and mass density characteristics of the void material are taken to be six orders of magnitude smaller than those of the solid material.

\section{1}

\section{The MBB beam problem}

In the examples that follow, variations of the so-called MBB beam compliance minimization problem are solved with a material usage constraint $50 \%$ that of the envelope structural volume. The problem is solved at a number of different mesh resolutions, both with and without perimeter constraints imposed. These examples were selected because they are representative of those in which "checkerboarding" material layout designs have been observed with Q4/U implementations.

A simply supported structural domain in which the structural material can be placed and to which a central point load is applied (Fig. 2a) has a length-to-height ratio of $6: 1$. The structural domain is first coarsely meshed with 60 by 10 square bilinear continuum elements, and the compliance minimization problem is solved with the Q4/Q4 implementation, resulting in the design shown in Fig. 2b. This material layout solution is significant in that it shows both a "layering" and "islanding" of black and white regions in the interior domain of the beam, which is quite difficult to interpret from a design perspective. (a)

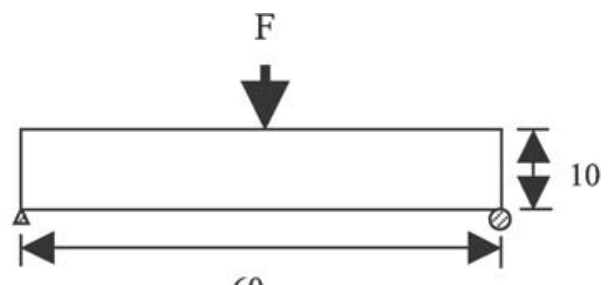

10

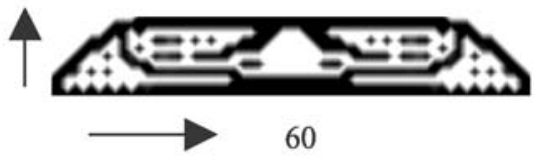

(e) $\pi=7.40$

30

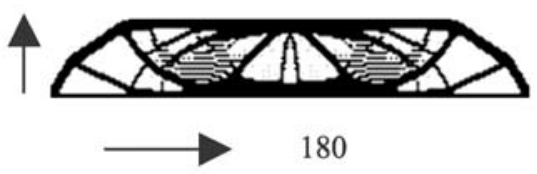

(f) $\pi=5.88$

50

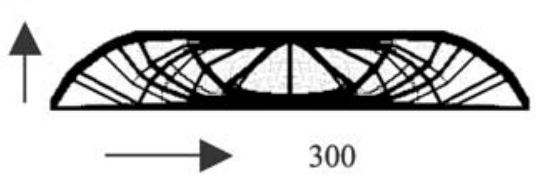

(g) $\pi=5.35$
10

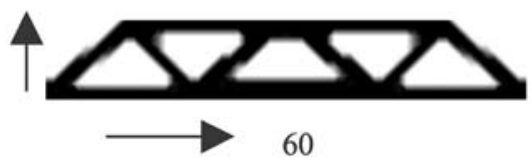

(h) $\pi=6.57$ 30

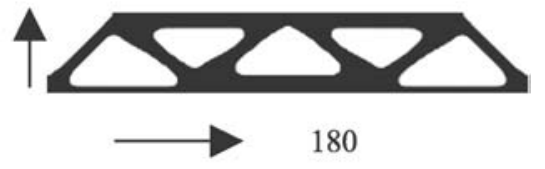

(i) $\pi=6.03$

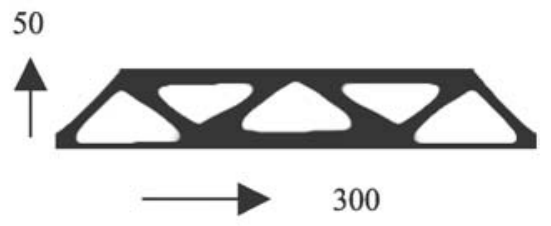

(j) $\pi=6.15$

Fig. 2 The MBB beam problem: (a) the design domain and the boundary conditions with a structural aspect ratio of $6 \times 1$; (b)-(d) the resulting designs in which mesh refinement through the thickness of the beam is progressively increased; (e)-(g) designs in which the mesh is uniformly refined in both directions; and $(h)-(j)$ designs with uniform mesh refinement and a perimeter constraint enforced 
The same finite element model for the beam was then progressively refined only in the vertical (layering) direction, with the respective solutions shown in Fig. 2c and $\mathrm{d}$ featuring progressively more mitigated layering and islanding.

As an alternative to selective refinement only in the vertical direction, the finite element model of the 6 by 1 structural domain was also refined uniformly in both directions to finer and finer meshes. The resulting layout solutions are shown in Fig. 2e-g. While these uniformly refined meshes also show layout solutions with increasingly mitigated layering/islanding, they also feature non-convergent grillage type design solutions. To ensure convergence of the material layout solution with mesh refinement, the compliance minimization problem was solved yet again with uniformly and progressively refined meshes and an imposed perimeter constraint of $p \leq 2(l+h)$, where $l$ is the width of the beam and $h$ is its vertical thickness or height. The layout solutions obtained with coarse and progressively finer meshes are shown in Figs. 2h-j. None of these designs feature any layering, and the design achieved with the coarse mesh appears virtually identical to that achieved with the much finer meshes. This is facilitated in the graphical display by capturing the spatial variation of design variables in each element instead of displaying each element with a uniform shade of gray corresponding to element-averaged design variable values. In this way, the Q4/Q4 implementation permits the edges of structural members in the layout design to slice through individual finite elements.

\section{2}

\section{The canyon bridge problem}

Grillage designs feature networks of slender structural members that continue to multiply with increasing mesh refinement. Since they are mesh-dependent and nonconvergent with mesh refinement, they are generally perceived as undesirable in continuum structural topology optimization. It is well established that such designs can be eliminated entirely by using spatial filtering of design variables and/or enforcing design perimeter constraints. On the other hand, in using continuum topology optimization to obtain concept designs of large-scale structures such as the bridge of this example, potentially important details can be lost by the application of these techniques. Since the Q4/Q4 implementation need not be used with methods that preclude possible checkerboarding, it can potentially capture some of these secondary details.

Here, optimization problems are solved to find optimal two-dimensional forms of a bridge to carry structural loads across a span of 1000 meters. A design traffic load of $10 \mathrm{kPa}$ is applied uniformly to the deck level of the bridge. The design domain that the bridge superstructure can potentially occupy is shown in Fig. 3a, and the structural material usage constraint is to be less than or equal to $12.5 \%$ of the bridge's envelope volume. In the first example, the design problem is solved to minimize structural compliance under the design traffic loading, and Fig. 3b shows the resulting material layout obtained with the Q4/Q4 implementation. Even though the secondary details of the material layout feature some grillage, the overall material layout is quite interpretable, and even the grillage members are suggestive of a system of tension members (cables) above the deck level and compression members beneath the deck.

The second optimization problem is solved to find the material layout that maximizes the minimum buckling eigenvalue of the structure. Important supporting details pertaining to both the formulation and solution of such stability optimization problems can be found in (Neves et al. 1995; Rahmatalla and Swan 2003a,b). A re-

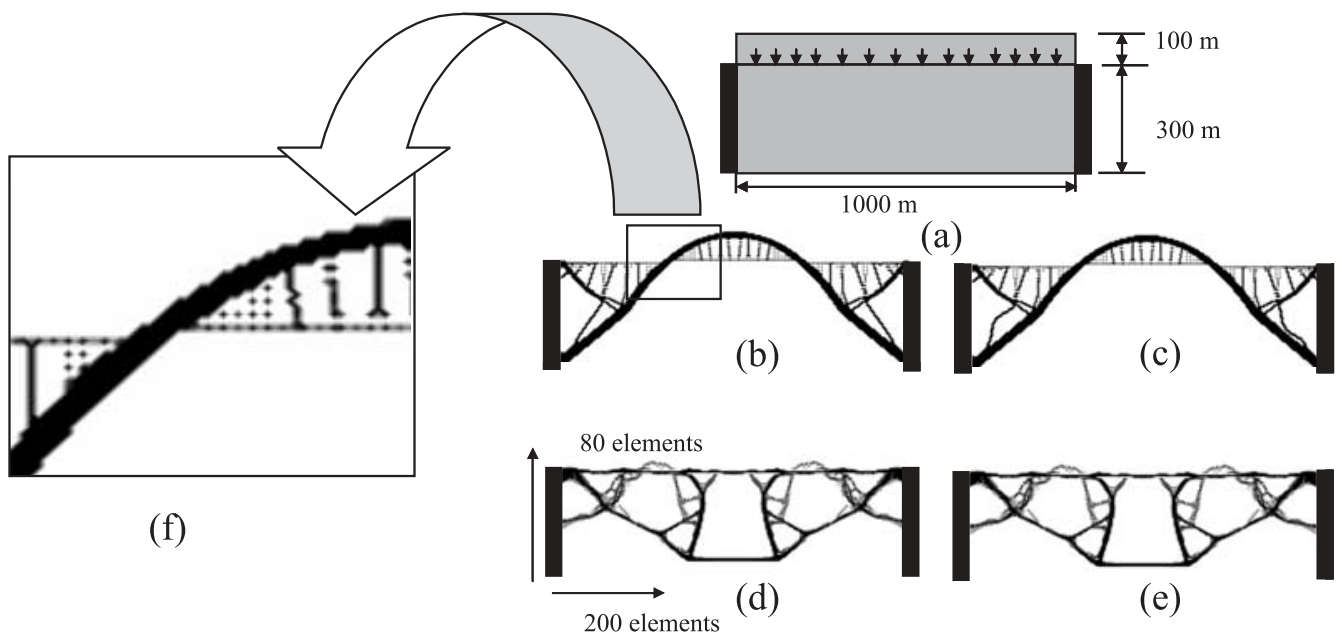

Fig. 3 Canyon bridge design problem: (a) the design domain and the boundary conditions with a uniformly loaded traction of $10 \mathrm{kPa}$; (b) the resulting material layout obtained by minimizing the elastic structural compliance; and (c) the buckling mode of this design; (d) the material layout obtained by maximizing the minimum critical buckling load with (e) the buckling mode which is localized and thus not visible; and (f) an expanded view of the layout design shown in (b) to show "layering" and "islanding" 
sulting optimal layout design for linearized buckling stability is shown in Fig. 3d. This material layout design is interpretable, avoiding buckling instabilities by using longer tension members and shorter, supported compression members.

\section{3}

\section{The problem of circle-domain design for stability}

In this example, a design load is applied to a circular design domain with fixed perimeter boundaries (Fig. 4a). The optimization problem is solved by placing a limited amount of structural material in the design domain in a way that achieves optimal structural performance. In Rahmatalla and Swan (2003b), a number of alternative problem formulations for obtaining sparse designs of optimum stability were presented and compared. Here, the intent is merely to demonstrate the characteristics of the Q4/Q4 topology implementation on this class of problems.

The first design problem solved is to minimize the generalized compliance of the structure up to the point of first instability, subject to a material usage constraint that is $2.5 \%$ of the envelope volume of the structure. The design solution obtained for this problem (Fig. 4b and c) employs both tension and compression members, and the material distribution is very clear, discrete, and free of any layering or islanding. The second design solution (Fig. 4d and e) is one obtained by maximizing the nonlinear buckling load magnitude, under a $2.5 \%$ material usage constraint. In this particular implementation, the design solution uses two struts that are primarily in tension. It too is very sharp, clear, and discrete, with no layering in the layout solution.

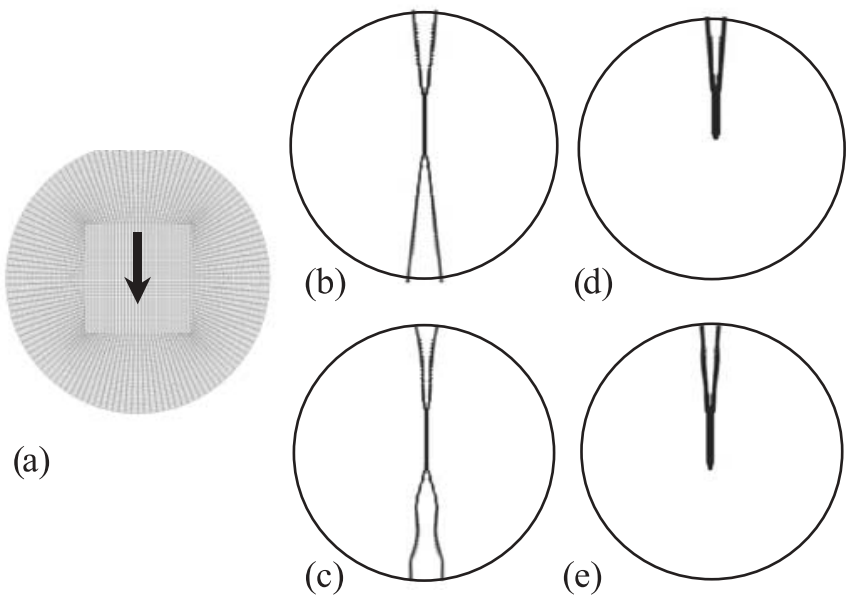

Fig. 4 Topological design solutions for the "circle-problem": (a) the design domain $\Omega$ and applied load (all nodes on the perimeter of $\Omega$ are fully restrained); (b) the design solution obtained by minimizing the elastic compliance up to a point of instability; (c) the deformed shape of design; and (d) the design solution obtained by maximizing the nonlinear buckling load, with (e) the deformed shape of the solution

\section{3}

\section{Discussion and conclusions}

The performance characteristics of a Q4/Q4 continuum topology optimization implementation based on nodal design variables have been explored on a number of test problems. The performance of the implementation on the test problems indicates that it is immune to the elementwise checkerboarding phenomenon, as expected, since it forces $\mathrm{C}^{0}$ continuity of the design variable field. Accordingly, the method does not require any assumption of filtering strength or length scales to preclude checkerboarding instabilities.

Although the present implementation is not susceptible to checkerboarding instabilities, it has been found in a limited number of instances to feature what might be an instability phenomenon characterized by "layering" or "islanding" of the design solutions. "Layering" and/or "islanding" designs appear to occur in cases where the mesh is marginally to insufficiently refined to capture the local material arrangements. Results of numerical experiments on the MBB beam examples appear to indicate, however, that, as the structural analysis mesh is progressively refined, the "layering" is strongly mitigated to the point of vanishing with sufficient refinement. Nevertheless, the numerical stability characteristics of "layering" or "islanding" in material layouts with the Q4/Q4 implementation warrant additional investigation.

For some classes of examples, especially those for the design of optimum buckling stability, the material layout designs show no propensity toward non-convergent grillage solutions. In certain classes of problems, however, especially those involving material layout optimization to minimize the compliance of structures undergoing flexure, the material layout design may not converge with mesh refinement. This non-convergence of designs with mesh refinement can occur with any of the implementations shown in Fig. 1. Accordingly, when employed on such problems, the Q4/Q4 implementation, like most all others, must be coupled with a method such as perimeter control that ensures convergence. When the Q4/Q4 implementation was used with meaningful perimeter constraints on $\mathrm{MBB}$ beam test examples to ensure design convergence, very sharp, interpretable designs were obtained even at fairly coarse levels of mesh resolution. That sharp interpretable designs can be achieved even at coarse mesh resolutions with the Q4/Q4 implementation is facilitated by its ability to resolve material interfaces that slice through individual finite elements.

Finally, although the proposed Q4/Q4 implementation has been tested herein using a volume-fraction (or density) continuum topology formulation, there is no reason that it cannot also be used successfully with homogenization-based continuum topology optimization methods, in which case microstructural design variables are spatially interpolated using nodal basis functions. 
Acknowledgements This research was funded in part by a grant from the University of Iowa CIFRE Program, and in part by NSF Grant No. DMS-9874015.

\section{References}

Bendsøe, M.P. 1989: Optimal shape design as a material distribution problem. Struct. Optim. 1, 193-202

Bendsøe, M.P.; Kikuchi, N. 1988: Generating optimal topology in structural design using a homogenization method. Comput. Methods Appl. Mech. Eng. 71, 197-224

Bruns, D.A.; Tortorelli, D.A. 1998: Topology optimization of geometrically nonlinear structures and compliant mechanisms. Comput. Methods Appl. Mech. Eng. 190, 3443-3459

Buhl, T.; Pedersen, W.; Sigmund, O. 2000: Stiffness design of geometrically nonlinear structures using topology optimization. Struct. Multidisc. Optim. 19, 93-104

Diaz, A.; Sigmund, O. 1995: Checkerboard patterns in layout optimization. Struct. Optim. 10, 40-45

Eschenauer, H.A.; Olhoff, N. 2001: Topology optimization of continuum structures: A review. Appl. Mech. Rev. 54(4), $331-390$

Fujii, D.; Kikuchi, N. 2000: Improvement of numerical instabilities in topology optimization using SLP method. Struct. Multidisc. Optim. 19, 113-121

Haber, R.B.; Jog, C.S.; Bendsøe, M.P. 1996: A new approach to variable-topology shape design using a constraint on perimeter. Struct. Optim. 11, 1-12

Jog, C.S.; Haber, R.B. 1996: Stability of finite element models for distributed-parameter optimization and topology design. Comput. Methods Appl. Mech. Eng. 130, 203-226
Neves, M.M.; Rodrigues, H.; Guedes, J.M. 1995: General topology design of structures with a buckling load criterion. Struct. Optim. 10, 71-78

Pedersen, N.L. 2000: Maximization of eigenvalues using topology optimization. Struct Multidisc Optim. 20, 2-11

Pedersen, C.B.W.; Buhl, T.; Sigmund, O. 2001: Topology synthesis of large-displacement compliant mechanisms. Int. J. Numer. Methods Eng. 50, 2683-2705

Poulsen, T.A. 2002: Topology optimization in wavelet space. Int. J. Numer. Methods Eng. 53, 567-582

Rahmatalla, S.F.; Swan, C.C. 2003a: Form-finding of sparse structures using continuum topology optimization. J. Struct. Eng. 129(12), 1707-1716

Rahmatalla, S.F.; Swan, C.C. 2003b: Continuum topology optimization of buckling-sensitive structures. AIAA J. 41(6), 1180-89

Sigmund, O.; Petersson, J. 1998: Numerical instabilities in topology optimization: A survey on procedures dealing with checkerboards, mesh-dependencies and local minima. Struct. Optim. 16, 68-75

Swan, C.C; Kosaka, I. 1997a: Voigt-Reuss topology optimization for structures with linear elastic material behaviors. Int. J. Numer. Methods Eng. 40, 3033-3057

Swan, C.C.; Kosaka, I. 1997b: Voigt-Reuss topology optimization for structures with nonlinear material behaviors. Int. J. Numer. Methods Eng. 40, 3785-3814

Swan, C.C.; Arora, J.S.; Kosaka, I.; Mijar, A.R. 1998: Concept design of bridges for stiffness and vibrations using continuum topology optimization. In: Srivastava, N.K. (ed.) Structural Engineering World Wide 1998. Proceedings of Structural Engineering World Congress (held in San Francisco) 